\title{
Platelet parameters and expression of platelet membrane glycoprotein in childhood acute lymphoblastic leukemia
}

\author{
Z. Huang, W.J. Liu, Q.L. Guo and C.Y. Liu \\ Department of Pediatrics, The First Affiliated Hospital of Sichuan Medical University, \\ Luzhou, Sichuan Province, China \\ Corresponding author: W.-J. Liu \\ E-mail: Iwejun_Iwj@163.com
}

Genet. Mol. Res. 14 (4): 16074-16089 (2015)

Received July 16, 2015

Accepted October 23, 2015

Published December 7, 2015

DOI http://dx.doi.org/10.4238/2015.December.7.20

\begin{abstract}
Platelet activation and functional changes were investigated in acute lymphoblastic leukemia (ALL) to provide the basis for early diagnosis and evaluation of curative effect. Platelet parameters, immature platelet fraction (IPF\%), immature platelet count (IPC), P-selectin (CD62p) (PAC-1) expression were detected in ALL, ALL-complete remission-induced (CR1), and normal groups with an automatic blood cell analyzer and flow cytometer. CD62 $p$ and PAC-1 were higher in the ALL group before adenosine-5diphosphate $(A D P)$ activation than in the normal group $(P<0.05)$; PAC-1 expression was higher and lower in the ALL-CR1 group than in normal and ALL groups $(P<0.05)$, respectively. CD62p and PAC-1 expression was lower in the ALL group than in the normal group after ADP activation ( $P<$ 0.05); PAC-1 expression was lower and higher in the ALL-CR1 group than in normal and ALL groups, respectively $(P<0.05)$. Platelet count $(P L T)$, mean platelet volume (MPV), platelet hematocrit (PCT), and platelet distribution width (PDW) were lower in the ALL group than in the ALL-CR1 group ( $<<$ 0.05). PLT, MPV, and PCT did not differ between the ALL-CR1 group and the normal group $(P>0.05)$. PDW did not differ statistically among all groups $(P>0.05)$. IPF\% and IPC values were higher and lower in the ALL group
\end{abstract}


than in normal and ALL-CR1 groups $(P<0.05)$, respectively. These did not differ significantly between the normal group and the ALL-CR1 group ( $P>$ 0.05). Therefore, ALL patients demonstrate platelet activation and platelet dysfunction; platelet parameters and membrane glycoprotein expression can be used to evaluate the effect of ALL.

Key words: Acute lymphoblastic leukemia; Platelet parameter; PAC-1; CD62p; Platelet function

\section{INTRODUCTION}

Acute lymphoblastic leukemia (ALL) is a neoplastic disease originating from B- or T-lymphocytes. In this disease, primitive cells proliferate abnormally, gather in the bone marrow, and inhibit normal hematopoiesis, leading to anemia, thrombocytopenia, and neutropenia; these primitive cells can also invade and cause lesions in extra-medullary tissues, such as the meninges, gonad, liver, spleen, and lymph node tissues. ALL accounts for $80 \%$ of childhood acute leukemia $(A L)$, and is the most common type of cancer affecting children. It is treated with a chemotherapybased comprehensive treatment program. The ALL prognosis has improved greatly over the past few years; however, only approximately $80-90 \%$ of the children achieve complete remission (Pui et al., 2011; Lohi et al., 2013). However, bleeding is known to occur during the course of diagnosis and chemotherapy, which is another important factor threatening the lives of ALL patients. The extent of reduction in the number of platelets is a determinant to assess the risk of bleeding. Patients with a low platelet count diagnosed and treated for a long period of time presented a very small amount of bleeding; however, patients with high platelet counts faced serious bleeding; therefore, bleeding was said to be associated with platelet function (Vinholt et al., 2014). Platelets have a short life span, and are not easily preserved; this hinders the clinical detection of platelet function. Therefore, the mechanism of change in platelet function under disease conditions is not fully understood.

Some of the platelet membrane glycoproteins (GP) are GP IIb/IIla and P-selectin. GP IIb/ IIla, the Fg-binding receptor mediated by platelets, is the most abundant glycoprotein in the platelet membrane. GP IIb/IIla binds to the exposed Fg receptor when vessel endothelium is damaged after collagen stimulation, leading to platelet aggregation. P-selectin is stimulated by a variety of factors that are quickly expressed on the platelet surface. Therefore, P-selectin is considered to be a specific and sensitive indicator of platelet activation. GP IIb/IIla and P-selectin can be used to assess drug efficacy and in disease prognosis. Reticulated platelets (RPs) are newborn peripheral blood platelets, which retain RNA in their cytoplasm. These are megakaryocytes in the immature stage that translate into platelets, and can be stained with fluorescent dyes. The monitoring of RP count in the chemotherapy and stem cell transplant process has far-reaching significance in the evaluation of the efficacy of therapeutic regimens, in assessing bone marrow function reconstruction, and in the prognosis of leukemia patients. It provides the therapeutic basis for determining the clinical applicability of prophylactic transfusion of single-donor platelets or megakaryocytestimulating factors (Xu and Liu, 2013). The level of expression of platelet membrane glycoprotein in cardiovascular disease is assessed by flow cytometry, in order to assess the platelet function, and to monitor the efficacy of anti-platelet therapy. The method has seen wide usage in clinical settings, and is considered to be an effective method for laboratory testing (Thomas et al., 2014). However, 
this method is rarely applied to the detection of platelet function in thrombocytopenia (Wang et al., 2013; Freitas et al., 2014; Montoro-García et al., 2014). In this study, we have researched the platelet function by analyzing the platelet parameters, platelet membrane glycoprotein expression, and the change in parameters of reticulated platelets. The determination of platelet parameters via a non-invasive examination is known to reflect the in vivo proliferation kinetics of platelets. It has a positive clinical significance in the early diagnosis, evolution, and evaluation of curative effect of platelets. In this study, flow cytometry was used to detect the platelet surface GP IIb/IIla, P-selectin, and RP content; in addition, a combined automated hematology analyzer was used to detect the platelet count (PLT), mean platelet volume (MPV), platelet hematocrit (PCT), and platelet distribution width (PDW). The peripheral platelet activation and reactivation, as well as the change in new synthetic platelets, in childhood ALL was assessed before and after chemotherapy. Further studies on the changes in platelet function during the course of diagnosis and after mitigation would provide the basis for early diagnosis, evolution, and evaluation of the curative effect of platelet therapy for ALL.

\section{MATERIAL AND METHODS}

\section{Materials}

\section{Main reagents and equipment}

Fluorescein-labeled platelet monoclonal antibody: isothiocyanate-labeled anti-platelet GP Ilb/Illa monoclonal antibody (PAC-1-FITC), phycoerythrin-labeled anti-platelet GMP-140 monoclonal antibody (CD62p-PE), peridinin chlorophyll protein-labeled anti-platelet GP IIla monoclonal antibody (CD61-PercP), phycoerythrin-labeled mouse IgG (MlgG-PE), CD62p-PE isotype control, phycocyanin-labeled anti-platelet GP Ib-ix monoclonal antibody (CD42b-APC), and $1.0 \mu \mathrm{g} / \mathrm{mL}$ thiazole orange (TO) were obtained from Sigma-Aldrich (St. Louis, MO, USA). RGDS (Arg-Gly-Asp-Ser) peptides (to be used as PAC-1 blockers) and synthetic arginyl-glycyl-aspartameseryl peptides were also obtained from Sigma-Aldrich. The remaining monoclonal antibodies were purchased from Becton-Dickinson and Company (USA). In addition, 0.2 M ADP (Sigma-Aldrich), $1 \%$ paraformaldehyde solution, phosphate-buffered saline (PBS), a BD FACSCantoTMII highspeed classifier flow cytometer (Becton-Dickinson and Company), and a BC-6800 automated hematology analyzer (Shenzhen Mindray) were used in this study.

\section{Sample collection}

All samples were obtained from patients (who signed informed consent forms, agreeing to participate in this study) treated in the Affiliated Hospital of the Luzhou Medical College from June 2012 to November 2013. The differences in age and gender among the three groups were not statistically significant $(P>0.05)$. The patients were classified into three groups: 1) ALL group [patients diagnosed by morphological analysis of bone marrow cells and MICM classification, who never received any related treatment, and did not display the presence of other neoplastic diseases, in line with the basic diagnostic criteria of ALL (Chinese Pediatric Society of Hematology, Chinese Medical Association, 2006)], comprising a total of 19 patients (11 males and 8 females), with the average age of $5.37 \pm 2.77$ years; the ALL-complete remission induced (ALL-CR1) group (ALL- 
CR1 standards in line with the efficacy standards of acute leukemia (Hu et al., 2002), containing 15 patients ( 7 males and 8 females), with an average age of $6.40 \pm 2.44$ years; and the control group (children selected from the pediatric surgery department prior to elective surgery, with normal blood routine, clotting, and liver and kidney function, and diagnosed a non-inflammatory disease after operation) comprising 15 patients (10 males and 5 females) with an average age of $4.73 \pm 1.98$ years (3 suffering from congenital torticollis, 2 diagnosed with varus, 9 with inguinal hernia, and 1 with cryptorchidism).

\section{Detection method}

The method prescribed by Wang (2005) was used in this study, as follows.

\section{Platelet membrane glycoprotein PAC-1 and CD62p detection}

Three milliliter of venous blood was extracted from the patients using a 5-mL syringe; $1.8 \mathrm{~mL}$ of the whole blood specimen was preserved in sodium citrate anticoagulant (mixed gently and allowed to rest) and immediately inspected. Freshly prepared ADP (50 $\mu \mathrm{L})$ was taken in a Falcon tube within $10 \mathrm{~min}$ after the addition of the blood sample; anticoagulant $(450 \mu \mathrm{L})$ was added to this tube, and the contents were gently vortexed, stamped, and incubated for $10 \mathrm{~min}$ at $37^{\circ} \mathrm{C}$ for platelet activation. The expression of PAC-1 and CD62p was measured by three-color flow cytometry. PE isotype control, CD61-PercP, and PAC-1-FITC (20 $\mu \mathrm{L}$ each) were added to the control tube; RGDS (10 $\mu \mathrm{L}), 20 \mu \mathrm{L}$ CD62p-PE, CD61-PercP, and PAC-1-FITC were added to $5 \mu \mathrm{L}$ inactive and activated whole blood (in separate experiment tubes; $N=2$ ). In addition, $5 \mu \mathrm{L}$ inactive whole blood was added to the control tube. The tubes were mixed gently and incubated for 20 min at room temperature in the dark. One milliliter $1 \%$ paraformaldehyde was added to each of these tubes; the tubes were mixed and incubated for $30 \mathrm{~min}$ in the dark at $2^{\circ}-8^{\circ} \mathrm{C}$. The samples were analyzed after $24 \mathrm{~h}$; the forward scatter, side scatter (SSC), and fluorescence signal were set for the flow cytometer; in addition, the CD61-PercP and SSC was set as the dual parameter and the isotype control tube was set as the negative group. Data was obtained for 10,000 platelets, and the positive expression rate of CD62p and PAC-1, and the mean fluorescence intensity (MFI) were calculated.

\section{Detection of platelet-related parameters (PLT, MPV, PDW, PCT, and RPs)}

Venous blood ( $4 \mathrm{~mL}$ ) was collected with a $5-\mathrm{mL}$ syringe, and stored in a tube containing the anticoagulant EDTA-K2; $2 \mathrm{~mL}$ of the whole blood specimen was preserved, and the remaining blood was retained in an automated blood cell analyzer to detect the platelet-related parameters. To the whole blood $(5 \mu \mathrm{L})$ present in the experimental tube, we added $5 \mu \mathrm{L}$ CD42b-APC and $50 \mu \mathrm{L}$ TO. The whole blood $(5 \mu \mathrm{L})$ present in the control tube was mixed with $5 \mu \mathrm{L}$ CD42b-APC and 50 $\mu \mathrm{L}$ PBS. These were mixed gently and incubated for $15 \mathrm{~min}$ at room temperature in the dark. One milliliter of $1 \%$ paraformaldehyde was added to each tube and mixed sufficiently; the tubes were then incubated at $2^{\circ}-8^{\circ} \mathrm{C}$. The samples were then analyzed within $45 \mathrm{~min}$. The CD42b-APC and SSC parameters were used to set the flow cytometer settings; the data was obtained for 10,000 platelets and the RP-positive expression rate (IPF\%) was computed. 


\section{Statistical analysis}

All data was analyzed using the SPSS (v.11.5) software (IBM, Armonk, NY, USA), and are reported as means \pm standard deviation (SD). Comparisons among groups were performed using one-way analysis of variance (ANOVA). Comparisons between two groups of data were performed using the LSD and rank-sum tests. $P$ values $\leq 0.05$ were considered to be statistically significant.

\section{RESULTS}

\section{Comparison of gender and age among the three groups}

A comparison of the gender and age among the members of the three groups has been summarized in Table 1. There were no significant differences about the gender and age of the patients among the three groups.

Table 1. Comparison of the various acute lymphocytic leukemia test groups and the normal control group based on the gender and age of the patients.

\begin{tabular}{|c|c|c|c|c|}
\hline \multirow[t]{2}{*}{ Group } & \multirow[t]{2}{*}{ Cases } & \multicolumn{2}{|c|}{ Gender } & \multirow[t]{2}{*}{ Age (means $\pm S D$ ) } \\
\hline & & Male & Female & \\
\hline ALL & 19 & 11 & 8 & $5.37 \pm 2.77$ \\
\hline ALL-CR1 & 15 & 7 & 8 & $6.40 \pm 2.44$ \\
\hline Normal & 15 & 10 & 5 & $4.73 \pm 1.98$ \\
\hline $\mathrm{F}$ & & \multicolumn{2}{|c|}{1.23} & 1.77 \\
\hline$P$ & & \multicolumn{2}{|c|}{$>0.05$} & $>0.05$ \\
\hline
\end{tabular}

\section{Detection of platelet parameters}

PLT, MPV, and PCT expression was found to be lower in the ALL group than in the normal and ALL-CR1 groups $(P<0.05)$. On the other hand, the expression of PLT, MPV, and PCT did not differ significantly between the ALL-CR1 and normal groups $(P>0.05)$. The expression of PDW also did not differ significantly among the three groups $(P>0.05$; Figure 1$)$.
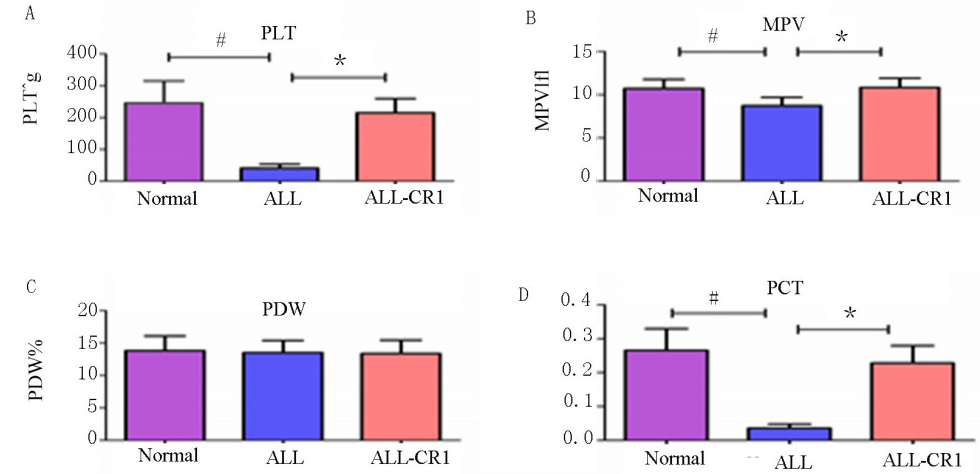

Figure 1. Expression of platelet indices. A. Expression of platelet count (PLT) among the three groups. B. Expression of mean platelet volume (MPV) among the three groups. C. Expression of platelet distribution widt (PDW) among the three groups. D. Expression of platelet hematocrit (PCT) among the three groups; ${ }^{\mathrm{P}}<<0.05$, for comparisons between the ALL group and the normal group; ${ }^{*} P<0.05$, when the ALL group was compared with the ALL-CR1 group. 


\section{Detection of the percentage and MFI of platelet membrane glycoprotein before and after ADP activation}

Before ADP activation, the percentage and MFI of CD62p and PAC-1 was found to be higher in the ALL group than in the normal and ALL-CR1 groups $(P<0.05)$; in addition, the percentage and MFI of PAC-1 was found to be higher in the ALL-CR1 group compared to that in the normal group $(P<0.05)$, and lower than that in the ALL group $(P<0.05)$. We observed no significant differences in the percentage and MFI of CD62p between the ALL-CR1 and normal groups $(P>0.05)$. Post-ADP activation, we observed that the percentage and MFI of CD62 $p$ and $P A C-1$ in the ALL group was lower than that of the normal and ALL-CR1 groups $(P<0.05)$, and that the percentage and MFI of PAC-1 in the ALL-CR1 group was lower than that of the normal group $(P<0.05)$ and higher than that of the ALL group $(P<0.05)$. We observed no significant differences in the percentage and MFI of CD62p between the ALL-CR1 group and the normal group ( $P>0.05)$ (Figures 2, 3, 4, 5, and 6).
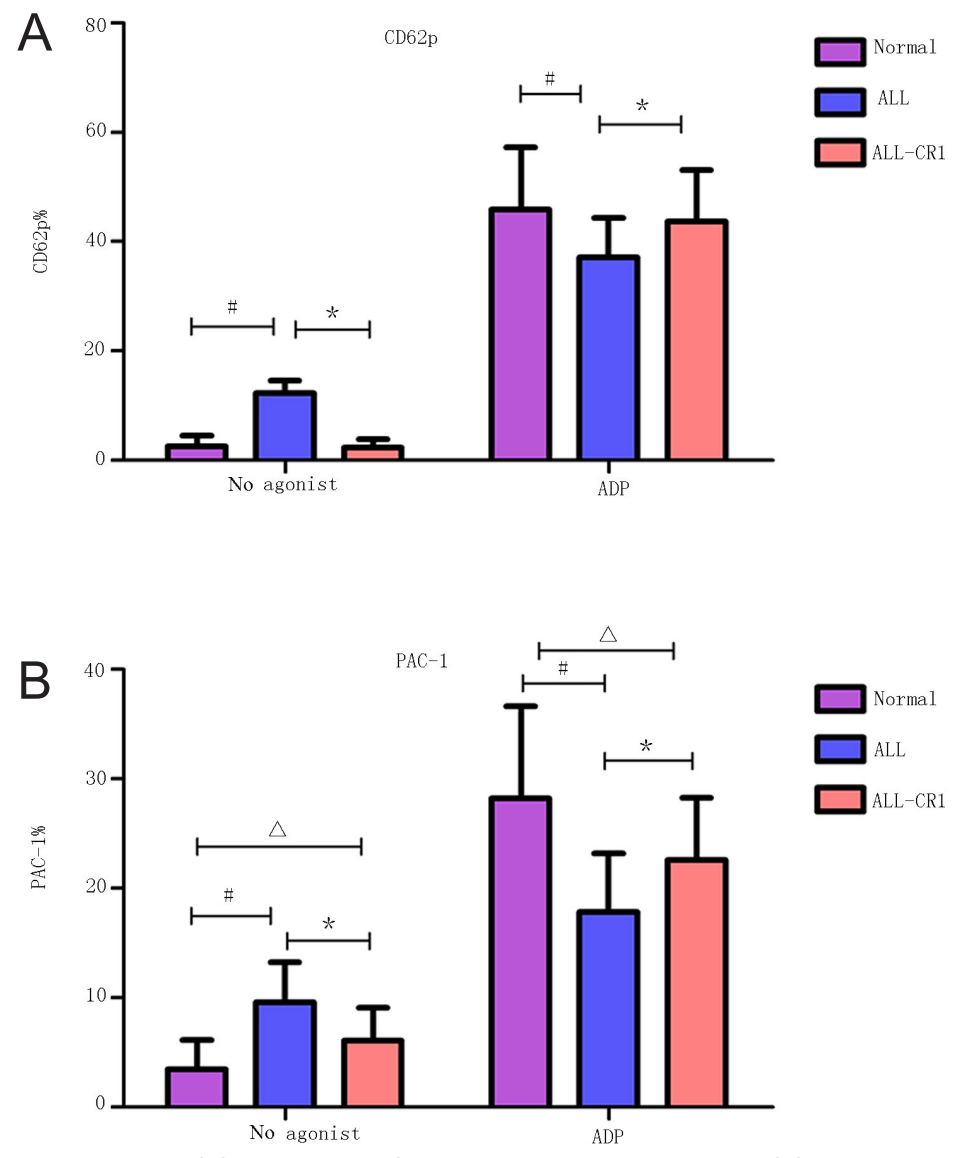

Figure 2. Percentage expression of CD62p and PAC-1. A. Percentage expression of CD62p in ALL, ALL-CR1, and normal groups before and after ADP activation. B. Percentage expression of PAC-1 among the three groups before and after ADP activation; ${ }^{*} P<0.05$, when the ALL group was compared with the normal group; ${ }^{*} P<0.05$, when the ALL group was compared with the ALL-CR1 group. ${ }^{\triangle} \mathrm{P}<0.05$, when the ALL-CR1 group was compared with the normal group. 


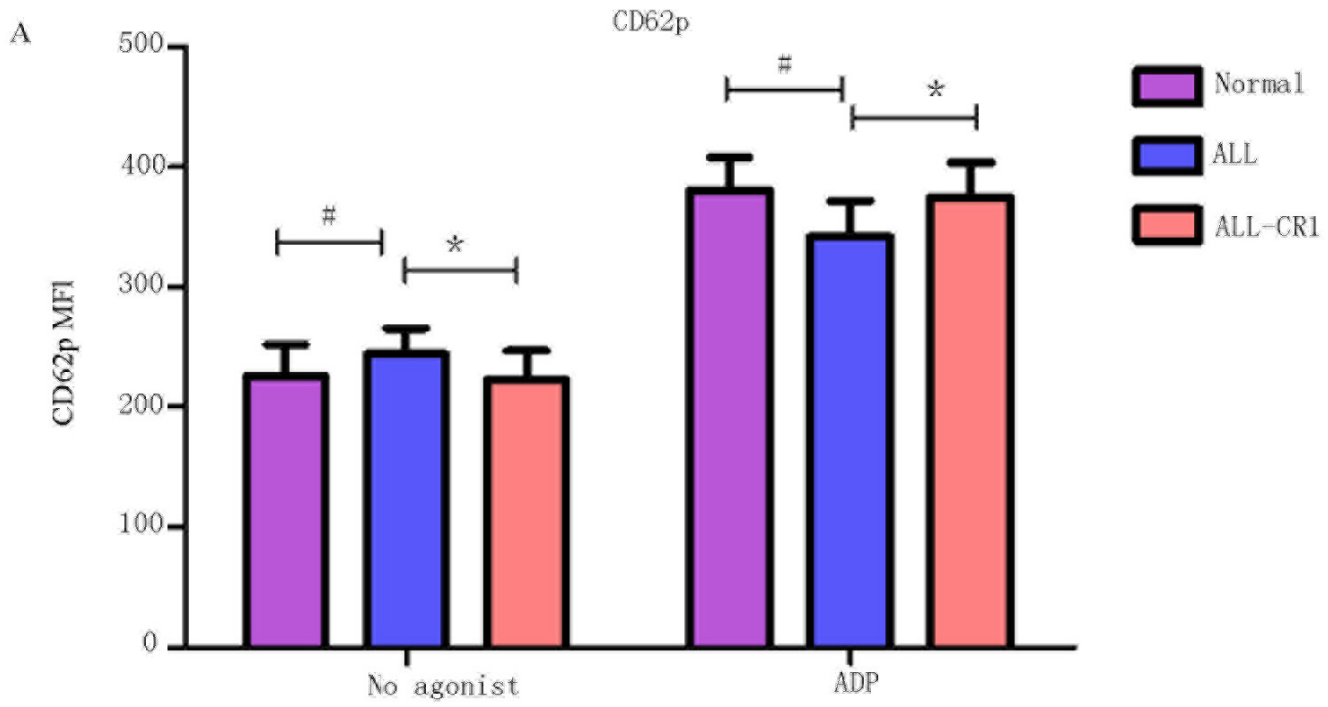

B

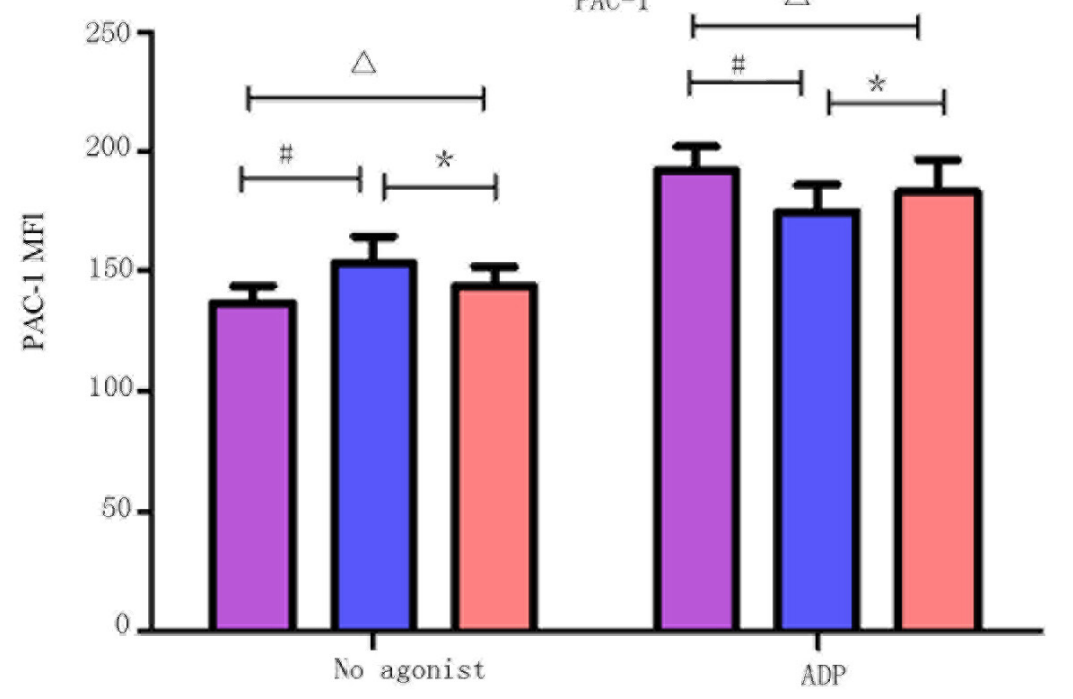

Figure 3. Expression of mean fluorescence intensity of CD62p and PAC-1. A. Expression of mean fluorescence intensity of CD62p in the three groups before and after ADP activation. B. Expression of mean fluorescence intensity of PAC-1 in the three groups before and after ADP activation; ${ }^{\# P}<0.05$ when the ALL group was compared with the normal group; * $P<0.05$, when the ALL group was compared with the ALL-CR1 group. ${ }^{\triangle} P<0.05$, when the ALL-CR1 group was compared with the normal group. 
A

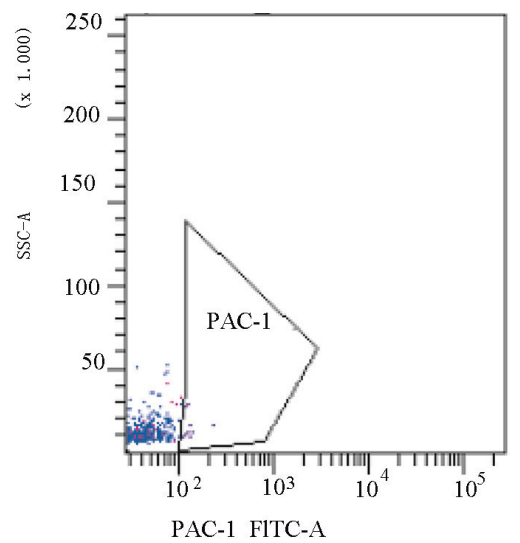

$\mathrm{B}$

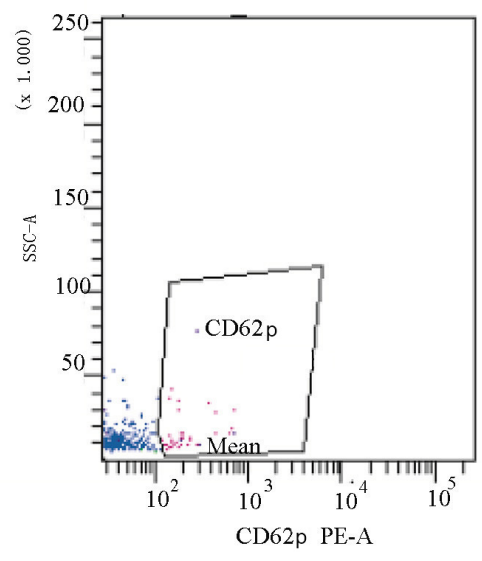

CD62p PE-A

\begin{tabular}{lrrll} 
Population & \#Events & \%Parent & Mean & Mean \\
\hline$\square$ P2 & 10,000 & 14.6 & 20 & 17 \\
PAC-1 & 90 & 0.9 & 131 & 146 \\
$\square$ CD62p & 374 & 3.3 & 51 & 259
\end{tabular}

$\mathrm{C}$

$\mathrm{D}$
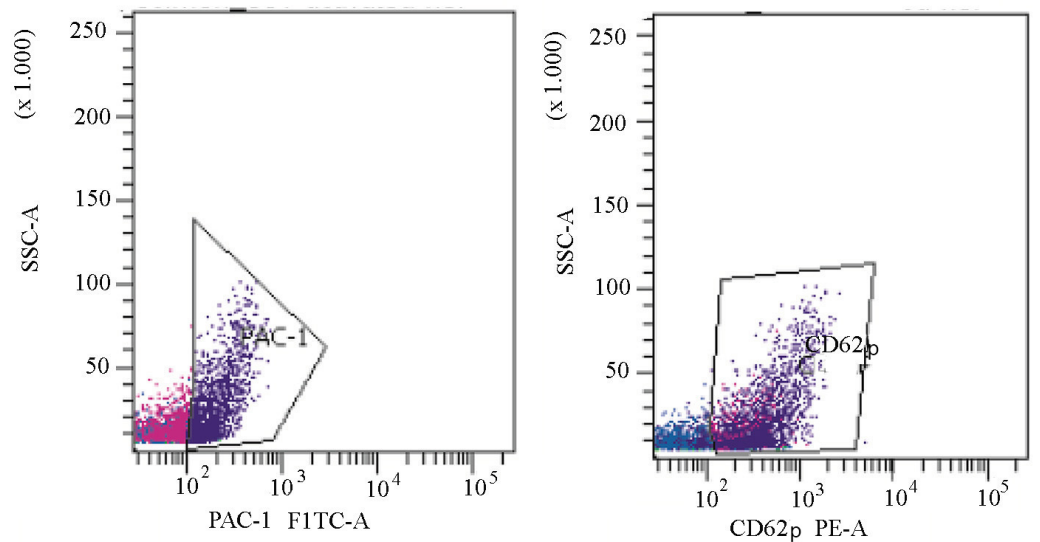

\begin{tabular}{lr} 
Population & \#Events \\
\hline$\square$ P2 & 10,000 \\
PAC-1 & 3,512 \\
CD62p & 5,271
\end{tabular}

Figure 4. PAC-1 and CD62p expression in the normal group before and after ADP activation. P2 represents the values for 10,000 platelets (events). A. Points in the polygon represent PAC-1 in the normal group before ADP activation. B. Points in the polygon represent CD62p in the normal group before ADP activation. C. Points in the polygon represent PAC-1 in the normal group after ADP activation. D. Points in the polygon represents CD62 $p$ in the normal group after ADP activation. 
A

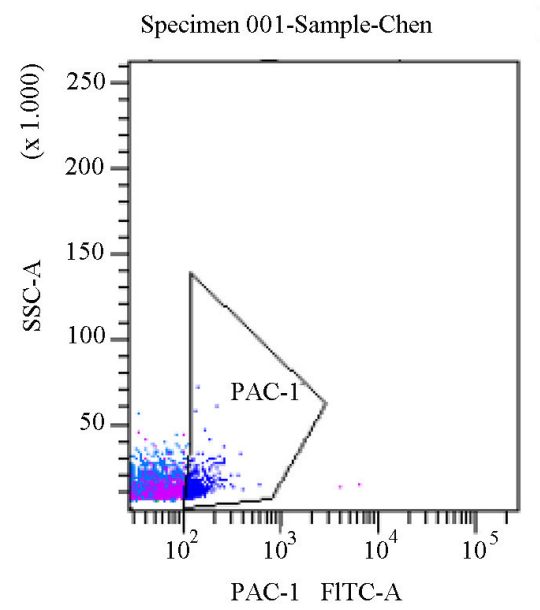

PAC-1 FITC-A
B

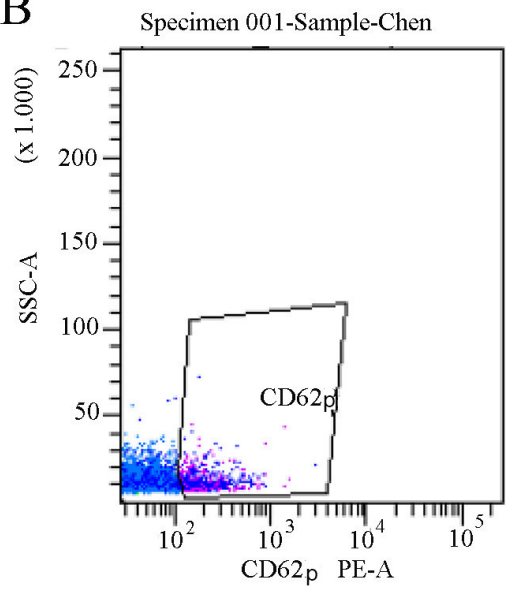

$\begin{array}{ccr}\text { \%Parent } & \text { Mean } & \text { Mean } \\ 39,5 & 29 & 33 \\ 5,4 & 136 & 134 \\ 7,6 & 89 & 230\end{array}$

$\mathrm{C}$

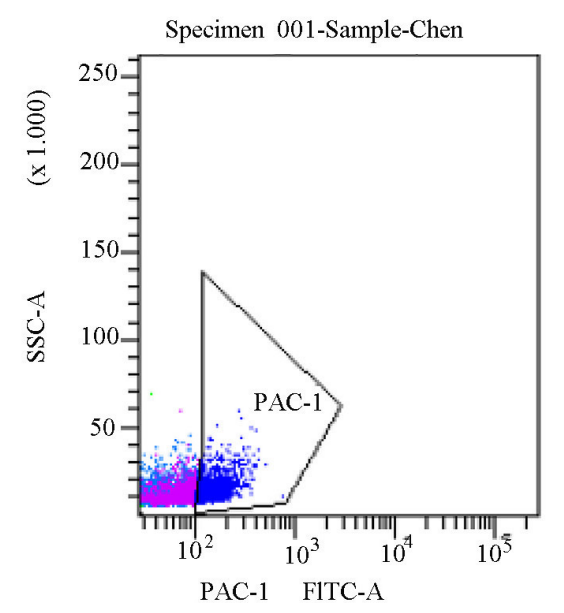

D Specimen 001-Sample-Chen

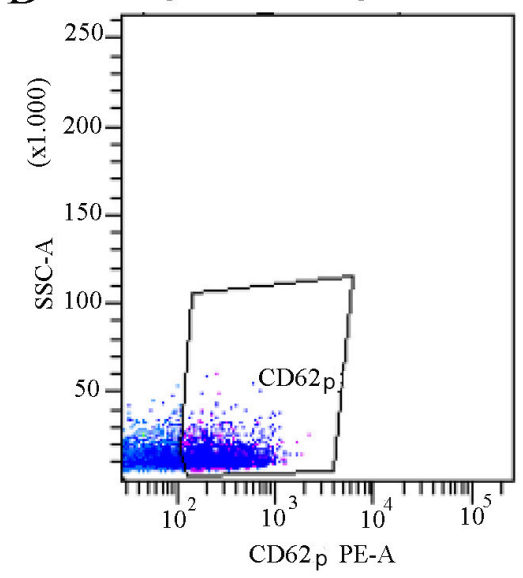

\begin{tabular}{lclcc} 
Population & \#Events & \%Parent & Mean & Mena \\
$\square$ P2 & 10,000 & 26,4 & 63 & 112 \\
\hline PAC-1 & 2,299 & 23,0 & 146 & 243 \\
$\square$ CD62.p & 3,129 & 31,3 & 106 & 294
\end{tabular}

Figure 5. PAC-1 and CD62p expression in the ALL group before and after ADP activation. P2 represents the values of 10,000 platelets (events). A. Points in the polygon represent PAC-1 in the ALL group before ADP activation. B. Points in the polygon represent CD62p in the ALL group before ADP activation. C. Points in the polygon represent PAC-1 in the ALL group after ADP activation. D. Points in the polygon represent CD62p in the ALL group after ADP activation. 

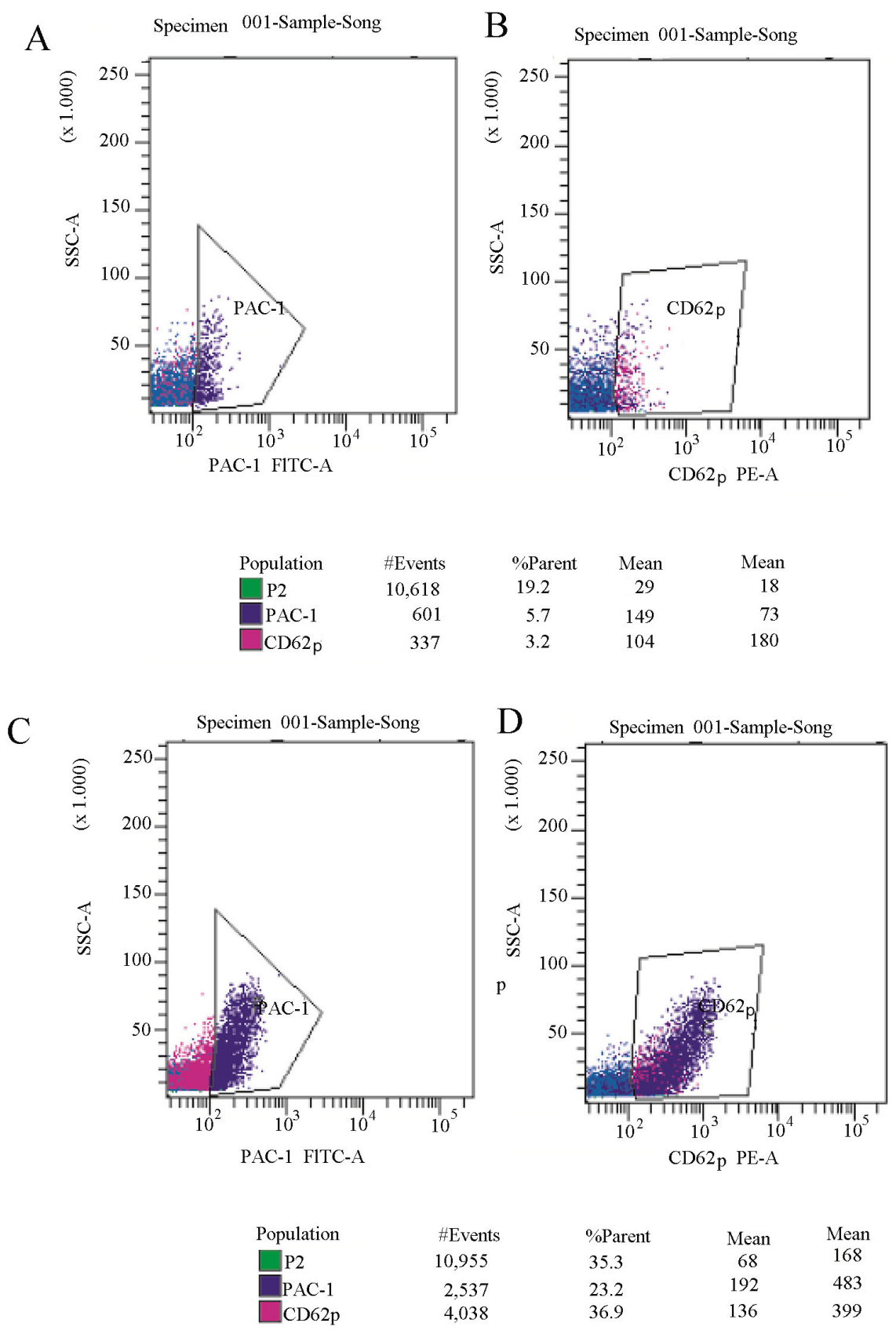

Figure 6. PAC-1 and CD62p expression in the ALL-CR1 group before and after ADP activation. P2 represents the obtained values for 10,000 platelets (events). A. Points in the polygon represent PAC-1 in the ALL-CR1 group before ADP activation. B. Points in the polygon represent CD62p in the ALL-CR1 group before ADP activation. C. Points in the polygon represent PAC-1 in the ALL-CR1 group after ADP activation. D. Points in the polygon represent CD62p in the ALL-CR1 group after ADP activation. 


\section{Detection of IPF\% and immature platelet count (IPC)}

The detection of IPF\% and IPC content in the three groups is summarized in Figures 7 and 8.
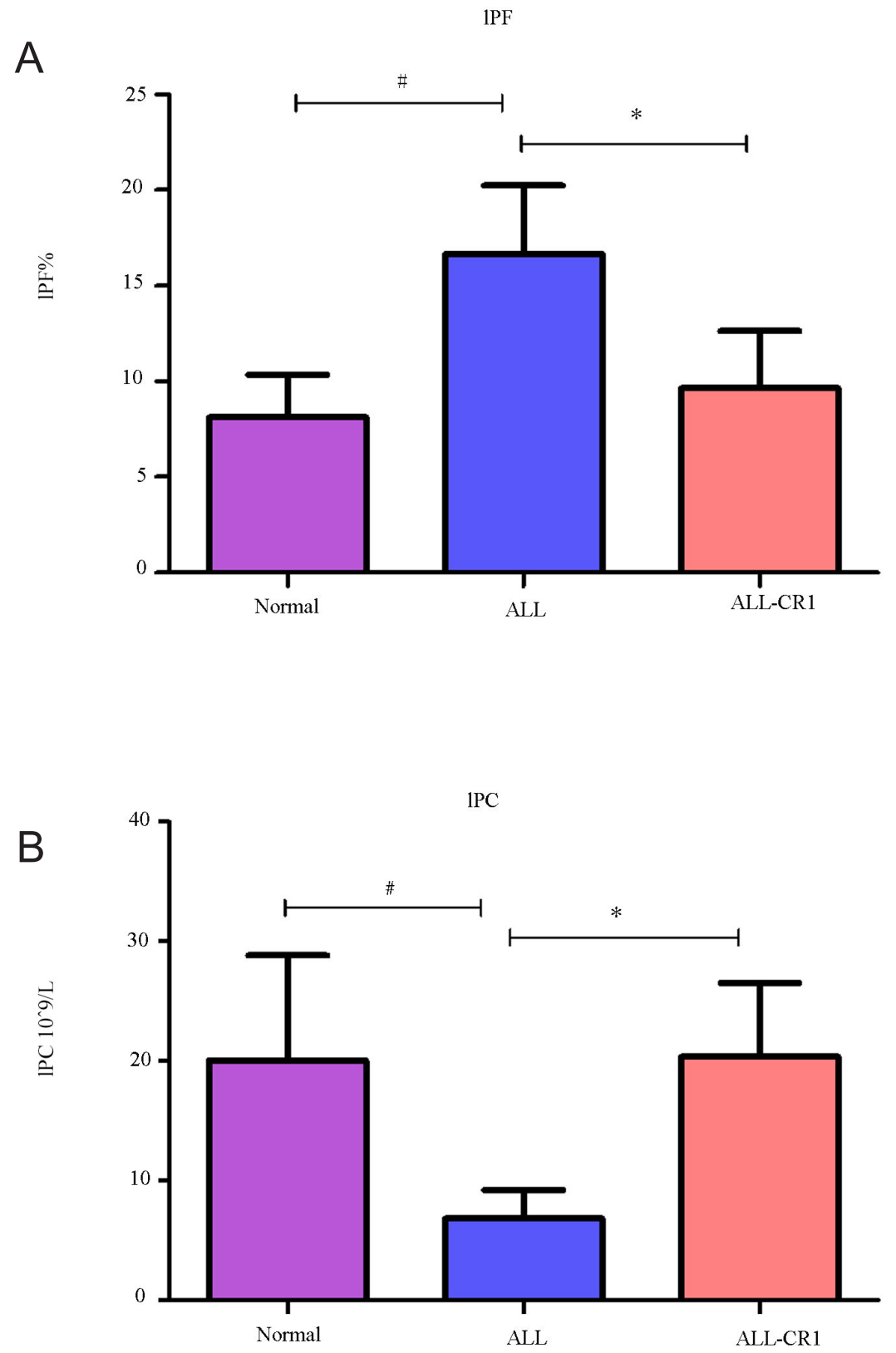

Figure 7. Expression of immature platelet fraction (IPF\%) and immature platelet count (IPC). A. Expression of IPF\% among the three groups. B. Expression of IPC among the three groups; ${ }^{P}<<0.05$, when the ALL group was compared to the normal group; * $P<0.05$, when the ALL group was compared to the ALL-CR1 group. 

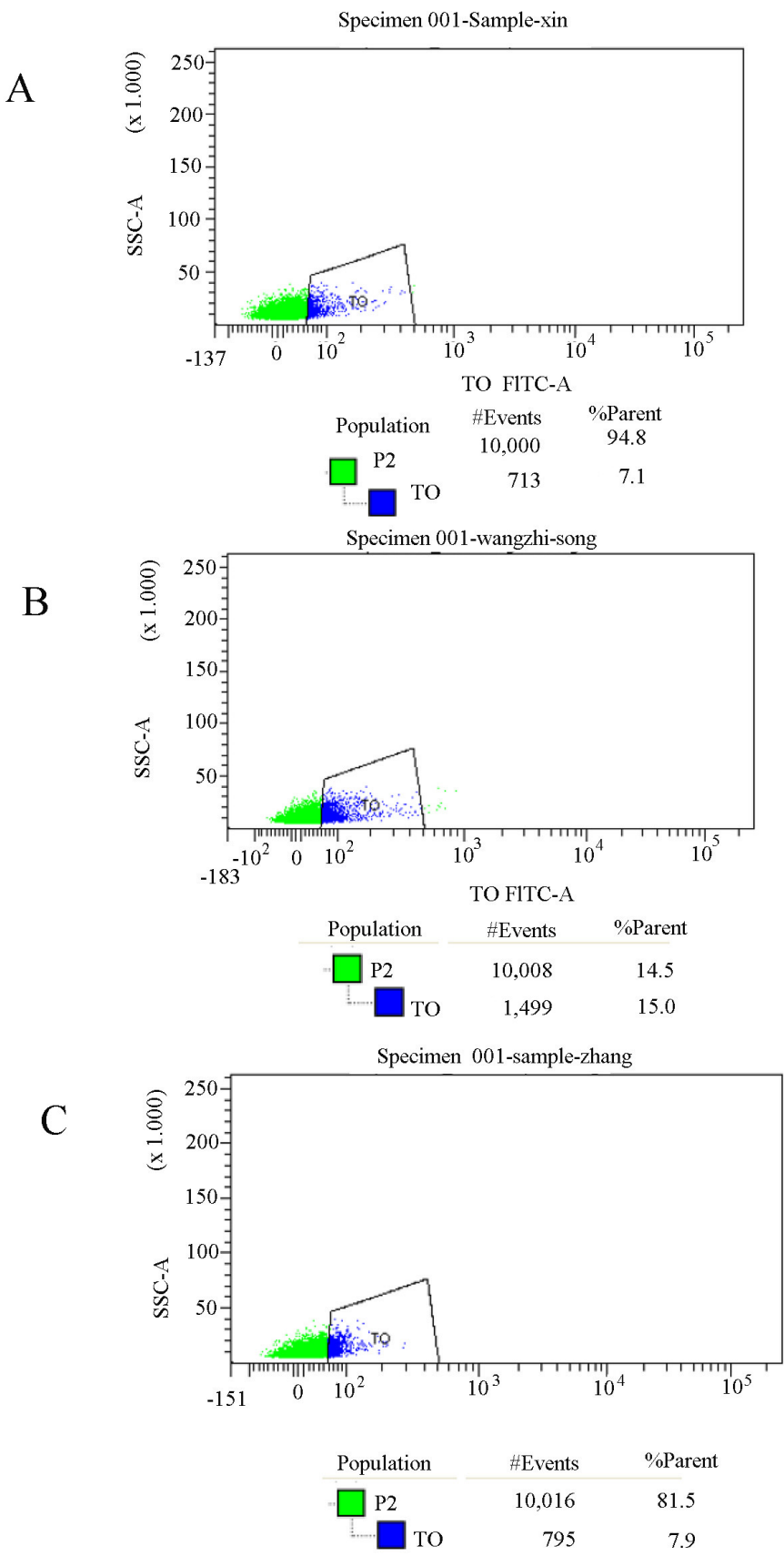

Figure 8. Expression of platelet fraction (IPF\%) in the platelets during the different stages of ALL, and in the normal group. P2 represents values obtained for 10,000 platelets (events); thiazole orange (TO) represents reticulated platelets. A. Points in the polygon represent IPF\% in the normal group. B. Points in the polygon represent IPF\% in the ALL group. C. Points in the polygon represent IPF\% in the ALL-CR1 group. 


\section{DISCUSSION}

Platelets are divided by the cytoplasm of megakaryocytes in the bone marrow. These are major, visible blood components that are involved in the processes of hemostasis and thrombosis. Under normal conditions, platelets exist as slightly convex discoids on both sides; the lipid membranes of platelets are studded with glycoproteins with multiple functionalities; the platelet membrane glycoprotein is the most abundant among these, and includes the GP Ib-IX-V, GP la/lla, GP VI, GP Ilb/llla, and P-selectin. They play a role in platelet activation, adhesion, and aggregation, and also participate in hemostasis and thrombosis.

GP IIb/IIla is the most abundant glycoprotein expressed on platelet membranes; the GP $\mathrm{Ilb} / \mathrm{Ill}$ a gene is located on the long arm of chromosome 17 (17q21-22). GP IIb/IIla expressed on the surface of resting platelets cannot be combined with $\mathrm{Fg}$; damage to the vascular endothelium results in platelet activation, which in turn leads to the expression of, and development of conformational changes in, the hidden GP IIb/IIla on the platelet membrane surface, which now combines with Fg to exert its physiological functions. GP IIb/IIla, combined with $\mathrm{Fg}$, is the final pathway of platelet aggregation (Sarratt et al., 2005). PAC-1 is a monoclonal antibody specific for the Fg binding site exposed after activation of the GP IIb/IIla on the platelet membrane surface, and can be used as a specific indicator of GP IIb/Illa activation.

P-selectin, also known as lysosomal membrane protein (CD62p) or granular membrane glycoprotein (GMP140), belongs to the selectin family of cell adhesion molecules. CD62p is rarely expressed during the quiescent stage. Platelet activation leads to the rapid fusion of CD62 $p$ with the plasma membrane, and subsequent expression on the platelet surface. The increase in CD62p is not reversed with the passage of time; therefore, CD62 $p$ is considered to be a major indicator (gold standard) of platelet activation (Mutlu et al., 2013).

In this study, the in vivo expression of CD62p and PAC-1 before ADP activation was taken to represent the platelet activation status, while the expression after ADP activation was concluded to represent the function of platelet activation. The expression of CD62 $p$ and PAC-1 in the ALL group was higher than that in the control group before ADP activation; this was observed to reduce gradually during remission. However, the PAC-1 expression did not return to normal. Platelet activation has been observed in various types of cancer, including colorectal, liver, breast, and lung cancer, and other malignant tumors; in addition, the prognosis was observed to worsen with the increase in platelet activation and aggregation, and the amount of transferred blood (Li et al., 2014; Mitrugno et al., 2014; Morimoto et al., 2014). In addition, we observed a significant increase in the CD62p and PAC-1 expression in a majority of cancer patients compared to the normal population, suggesting that CD62p and PAC-1 played an important role in tumor growth and metastasis. Gong et al. (2012) discovered a significant increase in the expression of CD62p in non-small cell lung cancer compared to the normal control group, indicating the capacity of CD62 $p$ to adhere to tumor cells, in order to facilitate its escape from mechanical damage caused by circulating blood. On the other hand, Bakewell et al. (2003) found that GP Ila/lllb played a key role in the process of tumor transfer to the bone tissue, suggesting that an anti-platelet GP Ila/IIlb drug could be used to prevent the transfer of tumor blood. These results strongly suggest that tumor cells induce platelet activation and aggregation. Children suffering from ALL showed the presence of a large number of leukemic cells in the blood and bone marrow; biologically active substances released by these leukemic cells have been shown to promote platelet activation and release. In addition, activated platelets are known to promote the adherence of leukemic cells to platelets, thereby mediating cell migration 
(Walenkamp et al., 2010; Sharma et al., 2014). The resulting CD62p and PAC-1 expression was observed to increase during the initial diagnosis of childhood ALL, demonstrating widespread infiltration. Some studies have indicated $(\mathrm{Xu}, 2005)$ that a reduction in the number of platelets leads to inflammatory mediators (released by tumor cells)-mediated endothelial cell damage. The initial diagnosis of childhood ALL often accompanies significant muco-cutaneous bleeding, which may be the cause of the increase in platelet activation. Recovery of bone marrow function in childhood ALL results in the return of CD62p expression to normal prior to ADP activation; however, the PAC-1 expression continues to increase. Despite clinical symptoms exhibiting complete remission of childhood ALL, a considerable number of leukemia cells are known to persist in vivo. These cells retain the capacity to damage vascular endothelial cells, interconnect with platelets, and subsequently stimulate platelet activation. ALL patients receive maintenance therapy even after complete remission; this may also result in enhanced platelet activation in vivo.

Childhood ALL often accompanies a reduction in platelet count. However, this reduction has so far not been fully correlated with the risk of bleeding and severity of the disease during clinical diagnosis and treatment (Huang and Liu, 2014). Previous studies have confirmed that defects in platelet function play an important role in leukemia bleeding (Slichter et al., 2010; Popov et al., 2014). In fact, Psaila et al. (2011) discovered a decrease in the expression of activationrelated receptors (GP IIb/IIla, CD62p, and GP Ib) on the surface of platelets obtained from acute myeloid leukemia or myelodysplastic syndromes (MDS) patients, as well as lower intrinsic activity and intrinsic platelet reactivity, compared to the corresponding values in samples obtained from idiopathic thrombocytopenic purpura patients. Sandes et al. (2012) used flow cytometry to detect the MDS platelet phenotype, and discovered changes in the platelet phenotype in a majority of the MDS patients. The results also indicated a lower expression of CD62p and PAC-1 in the ALL group after ADP activation; additionally, the levels of CD62p returned to normal during remission, while that of PAC-1 did not. This can be attributed to a number of factors: firstly, children diagnosed with ALL show reduced platelet counts and platelet dysfunction; the platelet function may significantly improve, but does not fully recover, after induction and remission. Sharma et al. (2011) revealed that the expression of the three major enzymes involved in energy metabolism (resulting in platelet dysfunction) during the initial or repeat diagnosis of various types of leukemia did not return to normal during remission. This suggested that leukemia platelet metabolism-related enzyme dysfunction reflected the genetic defects of megakaryocytes, while also explaining the reason for leukemia platelet dysfunction. Bone marrow megakaryocytes are inhibited or display functional defects in childhood ALL. In addition, the membrane proteins were of an abnormal quality in the produced platelets, leading to platelet dysfunction. The in vivo residual leukemic cells did not allow the bone marrow megakaryocytes to return to normal function during the remission period of childhood ALL, which explained the lack of correlation between the degree of bleeding and the platelet count.

PLT, MPV, PCT, and PDW are the major platelet parameters. PLT is a dynamic indicator of peripheral blood platelet production and death. The initial diagnosis of ALL shows a decrease in PLT, which returns to normal during remission. This may be because a large number of leukemic cells are involved in bone marrow infiltration, reduction of bone marrow function, decrease in platelet production, and bone marrow function recovery; the PLT is known to return to normal levels with remission. Low levels of PLT is known to indicate the complications of the disease and the indication of platelet transfusion. PCT is a product of PLT and MPV; therefore, changes in PCT are generally consistent with the changes in PLT. MPV reflects the proliferation and metabolism of 
megakaryocytes; platelet production, can be used as a reference to assess platelet function and identify the cause of thrombocytopenia (Balduini and Noris, 2013). PDW reflects the heterogeneity of platelet volume. Reduced MPV levels are observed during the initial diagnosis of childhood ALL $(P<0.05)$; these levels return to normal during remission. Differences in PDW are not statistically significant among the three groups $(P>0.05)$. MPV also reflects platelet enzymatic activity and functional status to some extent. As newborn platelets are bulky, the MPV is large, has an active function, and increases the rate and extent of $\mathrm{Fg}$ and thrombin-induced aggregation. Research has shown that reduced myeloproliferative function leads to a decrease in PLT, while MPV is reduced or remains unchanged (Chandra et al., 2010). Vinholt et al. (2014) suggested that the bleeding symptoms in thrombocytopenia patients may be related to a decrease in MPV. The results of this study indicated that childhood ALL incorporates thrombocytopenia and platelet dysfunction.

IPF\% and IPC reflect the megakaryocyte activity, identify thrombocytopenia caused by different reasons, and monitor the reconstruction of bone marrow function. The results revealed a significantly higher IPF\% and lower IPC in the ALL group compared to the normal group. There were no differences in IPF\% between the ALL-CR1 and normal groups $(P>0.05)$. As a result, we observed an increase in IPF\% in the ALL group, which was consistent with the results obtained by Strauss et al. (2011), who observed bone marrow megakaryocyte dysfunction, a shortened platelet life span, and a shorter life span of mature peripheral platelets compared to immature platelets, which could lead to a reduction in IPC and increase in IPF\%. Therefore, this study suggested that IPC was more meaningful in evaluating the efficacy of leukemia patients (compared to IPF\%), and assessing the bone marrow function reconstruction and prognosis. Have et al. (2013), who monitored the chemotherapy-induced decrease in platelet count and changes in IPF\% and IPC, found that the increase in IPC was 1-2 days earlier than the recovery of platelet count, while the changes in IPF\% were not obvious. Therefore, they concluded that IPC is a better indicator of bone marrow reconstruction during the predicted period of intensive treatment.

Future studies must analyze the platelet function from various angles, expanding the platelet biological function and the relation between platelet and disease, and exploring platelet parameters and the changes in IPF\%, IPC, and CD62p in childhood ALL during different periods. In addition, simpler means of platelet detection must be developed in the future, in order to find an effective method for disease diagnosis and prognosis. In addition, the mechanism of ALL platelet dysfunction must be elucidated to provide a theoretical basis for early clinical intervention for childhood ALL; in addition, methods must be developed for easy usage of clinical prophylactic platelet transfusions and megakaryocyte-stimulating factors.

\section{Conflicts of interest}

The authors declare no conflict of interest.

\section{REFERENCES}

Bakewell SJ, Nestor P, Prasad S, Tomasson MH, et al. (2003). Platelet and osteoclast $\beta 3$ integrins are critical for bone metastasis. Proc. Natl. Acad. Sci. U. S. A. 100: 14205-14210.

Balduini $\mathrm{CL}$ and Noris $\mathrm{P}$ (2013). Mean platelet volume for distinguishing between inherited thrombocytopenias and immune thrombocytopenia-response to Beyan. Brit. J. Haematol. 163: 413-414.

Chandra H, Chandra S, Rawat A and Verma SK (2010). Role of mean platelet volume as a discriminating guide for bone marrow disease in patients with thrombocytopenia. Int. J. Lab. Hematol. 32: 498-505.

Chinese Pediatric Society of Hematology, Chinese Medical Association (2006). The diagnosis and treatment recommendations of children with acute lymphoblastic leukemia (Third Revised Draft). Zhonghuaerkezazhi 44: 392-395. 
Freitas LG, Sathler-Avelar R, Vitelli-Avelar DM, Bela SR, et al. (2014). Preeclampsia: Integrated network model of platelet biomarkers interaction as tool to evaluate the hemostatic/immunological interface. Clin. Chim. Acta 436: $193-201$.

Gong L, Mi HJ, Zhu H, Zhou X, et al. (2012). P-selectin-mediated platelet activation promotes adhesion of non-small cell lung carcinoma cell on vascular endothelial cells under flow. Mol. Med. Rep. 5: 935-942.

Have LW, Hasle H, Vestergaard EM and Kjaersgaard M (2013). Absolute immature platelet count may predict imminent platelet recovery in thrombocytopenic children following chemotherapy. Pediatr. Blood Cancer 60: 1198-1203.

Hu YM, Jiang ZF and Zhu FT (2002). Text book of Pediatrics. 7th edn. People's Medical Publishing House, Beijing.

Huang Z and Liu WJ (2014). Progress in child thrombocytopenia platelet membrane glycoprotein. Zhonghuashiyongerkelinchuangzazhi 29: 227-230.

Li Y, Miao LY, Xiao YL, Cai HR, et al. (2014). Elevated platelets enhance cancer cell migration, promote hematogenous metastasis and associate with a poor prognosis in advanced non-small cell lung cancer cases. Asian Pac. J. Cancer Prev. 15: 139-143.

Lohi O, Kanerva J, Taskinen M, Harila-Saari A, et al. (2013). Childhood leukemia. Duodecim 129: 939-946.

Mitrugno A, Williams D, Kerrigan SW and Moran N (2014). A novel and essential role for FcyRII ${ }_{a}$ in cancer cell-induced platelet activation. Blood 123: 249-260.

Montoro-García S, Shantsila E, Hernández-Romero D, Jover E, et al. (2014). Small-size platelet microparticles trigger platelet and monocyte functionality and modulate thrombogenesis via P-selectin. Br. J. Haematol. 166: 571-580.

Morimoto Y, Nouso K, Wada N, Takeuchi Y, et al. (2014). Involvement of platelets in extra-hepatic metastasis of hepatocellular carcinoma. Hepatol. Res. 44: E353-E359.

Mutlu A, Gyulkhandanyan AV, Freedman J and Leytin V (2013). Concurrent and separate inside-out transition of platelet apoptosis and activation markers to the platelet surface. Br. J. Haematol. 163: 377-384.

Popov VM, Vladareanu AM, Bumbea H, Kovacs E, et al. (2014). Assessment of changes in membrane properties of platelets from patients with chronic myeloid leukaemia in different stages of the disease. Blood Coaqul. Fibrinolysis 25: 142-150.

Psaila B, Bussel JB, Frelinger AL, Babula B, et al. (2011). Differences in platelet function in patients with acute myeloid leukaemia and myelodysplasia compared to equally thrombocytopenic patients with immune thrombocytopenia. J. Thromb. Haemost. 9: 2302-2310.

Pui CH, Pei D, Campana D, Bowman WP, et al. (2011). Improved prognosis for older adolescents with acute lymphoblastic leukemia. J. Clin. Oncol. 29: 386-391.

Sandes AF, Yamamoto M, Matarraz S, Chauffaille Mde L, et al. (2012). Altered immunophenotypic features of peripheral blood platelets in myelodysplastic syndromes. Haematologica 97: 895-902.

Sarratt KL, Chen H, Zutter MM, Santoro SA, et al. (2005). GPVI and a2b1 play independent critical roles during platelet adhesion and aggregate formation to collagen under flow. Blood 106: 1268-1277.

Sharma D, Brummel-Ziedins KE, Bouchard BA and Holmes CE (2014). Platelets in tumor progression: a host factor that offers multiple targets in the treatment of cancer. J. Cell Physiol. 229: 1005-1015.

Sharma S, Purohit A, Pati HP and Kochupillai V (2011). Platelet enzyme abnormalities in leukemias. Indian J. Cancer 48: 323-327.

Slichter SJ, Kaufman RM, Assmann SF, McCullough J, et al. (2010). Dose of prophylactic platelet transfusions and prevention of hemorrhage. N. Engl. J. Med. 362: 600-613.

Strauss G, Vollert C, von Stackelberg A, Weimann A, et al. (2011). Immature platelet count: a simple parameter for distinguishing thrombocytopenia in pediatric acute lymphocytic leukemia from immune thrombocytopenia. Pediatr. Blood Cancer 57 : 641-647.

Thomas MR, Wijeyeratne YD, May JA, Johnson A, et al. (2014). A platelet P-selection test predicts adverse cardiovascular events in patients with acute coronary syndromes treated with aspirin and clopidogrel. Platelet 25: 612-618.

Vinholt PJ, Hvas AM and Nybo M (2014). An overview of platelet indices and methods for evaluating platelet function in thrombocytopenic patients. Eur. J. Haematol. 92: 367-376.

Walenkamp AM, Bestebroer J, Boer IG, Kruizinga R, et al. (2010). Staphylococcal SSL5 binding to human leukemia cells inhibits cell adhesion to endothelial cells and platelets. Cell Oncol. 32: 1-10.

Wang B, Peng Y, Dong J, Lin J, et al. (2013). Human platelets express functional thymic stromal lymphopoietin receptors:a potential role in platelet activation in acute coronary syndrome. Cell Physiol. Biochem. 32: 1741-1750.

Wang JZ (2005). Clinical flow cytometry. 1st edn. Shanghai Science and Technology Press, Shanghai, 401-402, 431-432.

Xu Q and Liu WJ (2013). Platelet changes in acute leukemia. Cell Biochem. Biophysics 67: 1437-1479.

Xu SX (2005). Progress in the role of platelet activation in inflammation. Guojijianyanyixuezazhi 8: 542-543. 